\title{
Apicificação em dente imaturo traumatizado com fratura corono-radicular: relato de
}

\section{caso}

\author{
Apixification on immature tooth traumatized with crown-root fracture: case report
}

Apexificación en diente inmaduro traumatizado con fractura coronal-radicular: reporte de caso

Recebido: 13/01/2022 | Revisado: 18/01/2022 | Aceito: 25/01/2022 | Publicado: 26/01/2022

Thatiana Cristino Freire

ORCID: https://orcid.org/0000-0002-8608-7324 Universidade Federal de Alagoas, Brasil

E-mail: thatianafreire@ hotmail.com

Andréa Albuquerque Lima

ORCID: https://orcid.org/0000-0001-5861-3799

Universidade Federal de Uberlândia, Brasil

E-mail: dra.andrealima@gmail.com

Kaio Coura Melo Pacheco

ORCID: https://orcid.org/ 0000-0001-7804-5426 Universidade Federal de Alagoas, Brasil

E-mail: kaio.melo_95@hotmail.com

Phillip Lucas Ricardo Pereira

ORCID: https://orcid.org/0000-0003-0911-4672 Faculdade Herrero, Brasil

E-mail: phillippereira@gmail.com

Clóvis Stephanno Pereira Bueno

ORCID: https://orcid.org/0000-0001-9149-1674 Faculdade Herrero, Brasil

E-mail: clovisspbueno@gmail.com

Daniel Pinto de Oliveira

ORCID: https://orcid.org/0000-0003-1992-9920 Universidade Federal de Alagoas, Brasil

E-mail: dpoendo@yahoo.com.br

Leopoldo Cosme Silva

ORCID: https://orcid.org/0000-0002-5755-1933 Universidade Federal de Alagoas, Brasil

E-mail: leopoldocosme@gmail.com

Laís Christina Pontes Espíndola ORCID: https://orcid.org/0000-0002-1023-7891 Universidade Federal de Alagoas, Brasil

E-mail:laisespindola@hotmail.com

Dyana dos Santos Fagundes

ORCID: https://orcid.org/0000-0002-7427-4114

Universidade Federal de Alagoas, Brasil

E-mail: dydyana_fagundes@hotmail.com

\section{Resumo}

Os traumatismos dentários constituem um problema de saúde de alta frequência em crianças e adultos jovens, e estão associados a consequências psicológicas, funcionais e ainda estéticas. A necrose pulpar é uma das principais consequências após o trauma dental, implicando, dessa forma, na falha do desenvolvimento radicular dos dentes permanentes recém erupcionados, os quais em razão disso permanecem com paredes finas e ápice aberto. O objetivo deste trabalho foi descrever uma opção de tratamento que permite, ao mesmo tempo, o fechamento do ápice radicular e o reforço das paredes apicais em dentes imaturos que apresentem status de necrose pulpar após esses sofrerem trauma. Foi utilizado como técnica a apicificação com aplicação única de hidróxido de cálcio. O presente trabalho permitiu concluir que a apicificação com hidróxido de cálcio é uma alternativa de baixo custo, facilmente aplicável na rotina clínica e que apresenta altas taxas de sucesso no tratamento de dentes imaturos traumatizados.

Palavras-chave: Endodontia; Endodontia regenerativa; Necrose pulpar; Hidróxido de cálcio; Trauma dental.

\begin{abstract}
Dental trauma is a health problem with high frequency in children and young adults. They are associated to psychological, functional and aesthetics consequences. Pulp necrosis is one of the main consequences after a dental trauma and implies failure in the root development of newly erupted permanent teeth, which therefore remain thin
\end{abstract}


walls and open apex. The objective of this study was to describe a treatment option that allows the development of the root apex and the strengthening of the apical walls in immature teeth that present pulp necrosis after trauma. Apexification with a single application of calcium hydroxide was used as technique. This study concluded that calcium hydroxide apexification is a low cost alternative, easily applicable in the clinical routine and that presents high success rates in the treatment of immature traumatized teeth.

Keywords: Endodontics; Regenerative endodontics; Dental pulp necrosis; Calcium hydroxide; Dental trauma.

\section{Resumen}

El trauma dental es un problema de salud de alta frecuencia en niños y adultos jóvenes, y está asociado a consecuencias psicológicas, funcionales y estéticas. La necrosis pulpar es una de las principales consecuencias tras el traumatismo dental e implica un fallo en el desarrollo radicular de los dientes permanentes recién erupcionados, que por ello quedan con paredes delgadas y ápice abierto. El objetivo de este estudio fue describir una opción de tratamiento que permita, al mismo tiempo, el cierre del ápice radicular y el refuerzo de las paredes apicales en dientes inmaduros que presentan un estado de necrosis pulpar posterior a un traumatismo. Se utilizó como técnica la apexificación con una sola aplicación de hidróxido de calcio. Este estudio permitió concluir que la apexificación con hidróxido de calcio es una alternativa de bajo costo, fácilmente aplicable en la rutina clínica y que tiene altas tasas de éxito en el tratamiento de dientes inmaduros traumatizados.

Palabras clave: Endodoncia; Endodoncia regenerativa; Necrosis de la pulpa dental; Hidróxido de calcio; Trauma dental.

\section{Introduçãa}

Os traumatismos dentários constituem um problema de saúde que está ligado a consequências estéticas, funcionais e psicológicas (Zengin, Celenk, Sumer \& Cankaya, 2015). Ocorrem com grande frequência em crianças na fase pré-escolar e em jovens adultos, sendo as fraturas coronárias o tipo de traumatismo mais frequente na dentição permanente, acometendo principalmente o incisivo central superior (Shabahang, 2013).

A depender do estágio de desenvolvimento da raiz, o dente pode apresentar canais amplos, paredes dentinárias finas e ápices abertos, o que os torna mais propensos a fraturas (Vellore, 2010), ou apresentar espessura de paredes adequada, porém ápice aberto. Essa diferença do padrão radicular pode ser justificada devido aos dentes permanentes levarem aproximadamente três anos para terem seu desenvolvimento completo após erupção (Lopes, Siqueira Junior \& Estrela, 1999; Shaik et al., 2021). Dentes com rizogênese incompleta, que necessitam de tratamento endodôntico após trauma ou exposição por cárie, seguem os mesmos princípios aplicados no tratamento endodôntico de dentes completamente desenvolvidos (Lopes, Siqueira Junior \& Estrela, 1999; Vellore, 2010). No entanto, o objetivo é mais complexo, pois busca-se a continuação do desenvolvimento radicular nos casos de polpa vital (apicegênese) e em casos de necrose da polpa, a revascularização pulpar ou a completa formação do ápice radicular através de tecido duro calcificado (apicificação) (Lopes, Siqueira Junior \& Estrela, 1999), sendo essa última técnica o enfoque deste trabalho.

Quando o dente imaturo que apresenta ápice aberto e espessura de paredes dentinárias suficientemente desenvolvidas é acometido por trauma ou cárie, levando a necrose da polpa, o tratamento de escolha é a apicificação. Trata-se de um método não cirúrgico em que um material é introduzido no interior do canal e induz a continuação da formação do ápice radicular por deposição de tecido duro (Damle et al., 2016) facilitando a adaptação dos materiais durante a obturação do canal (Lopes, Siqueira Jr. \& Neves, 2015).

Nos últimos anos, tem havido um interesse considerável a respeito da utilização de fatores de crescimento e moléculas bioativas no tratamento conservador pulpar e na apicificação (Lopes, Siqueira Junior \& Estrela, 1999). Várias substâncias foram utilizadas com o objetivo de se obter o completo desenvolvimento do ápice radicular (Lopes, Siqueira Junior \& Estrela, 1999), sendo o hidróxido de cálcio e o MTA os principais materiais utilizados para esse fim. Porém, o hidróxido de cálcio tem sido o principal material de escolha e de maior suporte científico no tratamento endodôntico de dentes com rizogênese incompleta (Lopes, Siqueira Junior \& Estrela, 1999; Shaik, 2021). A apicificação pode ser realizada sob duas técnicas: mediata ou imediata. 
A apicificação mediata é realizada através de trocas periódicas de hidróxido de cálcio. Devido aos seus diversos efeitos biológicos, o tempo necessário para a apicificação completa utilizando $\mathrm{Ca}(\mathrm{OH})_{2}$ foi relatado como variável, levando de 5 a 20 meses (Damle et al., 2016). Enquanto na apicificação imediata, logo após o preparo e desinfecção do canal radicular, utiliza-se como material obturador permanente do segmento apical radicular (tampão apical) uma pasta à base de hidróxido de cálcio ou o MTA. Esse tampão apical, além de servir como barreira mecânica, estimula o fechamento apical e atua como barreira física no ápice radicular permitindo a adaptação do material obturador (Lopes, Siqueira Jr. \& Neves, 2015).

O MTA vem sendo utilizado com grande frequência na técnica imediata por se constituir de um material com citotoxicidade mínima, baixo grau de inflamação, indução de tecido duro nos tecidos periodontais e capacidade superior de vedação através de adaptação marginal aprimorada, além de um menor tempo de tratamento requerido (Boufdil, Mtalsi, El Arabi \& Bousfiha, 2020; Damle et al., 2016; Shaik et al., 2021). As desvantagens do MTA estão relacionadas à ausência de reforço das paredes dentinárias, seu alto custo, possibilidade de escurecimento dentário, facilidade de manipulação diminuída em relação ao hidróxido de cálcio, além da pequena quantidade de estudos que avaliam seu comportamento à longo prazo em dentes permanentes jovens (Boufdil, Mtalsi, El Arabi \& Bousfiha, 2020; Kandemir Demirci, Kaval, Güneri \& Çalışkan, 2020; Lin et al., 2016; Refaei, Jahromi \& Moughari, 2020).

A apicificação por troca de hidróxido de cálcio é a técnica mais usualmente praticada por se tratar de um material que não apresenta reações periapicais, tem resultados previsíveis, permite a adição de outras substâncias e por ser uma técnica fácil e de baixo custo (Damle et al., 2016) com uma taxa de sucesso variando entre 71\% (Botero, 2017) a 100\% (Dominguez Reyes, Munoz Munoz, \& Aznar Martin, 2005). A utilização do hidróxido de cálcio no interior do canal de um dente com ápice aberto provoca uma resposta semelhante à quando é utilizado em contato com a polpa coronária. Porém, ao invés de formar dentina, um tecido cementário ou de reparação é formado, induzindo o desenvolvimento completo do ápice radicular (Harlamb, 2016).

Desta forma, o objetivo deste trabalho foi descrever um caso clínico, envolvendo o tratamento endodôntico e apicificação com hidróxido de cálcio, em dente imaturo após histórico de trauma. O presente caso foi realizado com embasamento teórico através da revisão de literatura e de estudos de casos clínicos presentes na base de dados Pubmed.

\section{Metodologia}

Para o desenvolvimento deste trabalho, foi selecionado um caso clínico de trauma corono-radicular, 15 dias após sofrer queda da própria altura, com trauma na região de incisivos superiores, ocasionando a fratura do elemento 11. Foi realizada a anamnese do paciente, para a coleta do maior número de informações a respeito do trauma e todas as condutas que foram tomadas no momento e após o acontecimento do mesmo. Foi entregue ao responsável do paciente um termo de consentimento livre e esclarecido, para a ciência do mesmo sobre a condução do caso, e os riscos que envolvem o procedimento. Foi realizado um termo de consentimento livre e esclarecido, para que o paciente tomasse ciência de como seria conduzido seu caso, bem como os riscos que envolvem este tipo de trauma. O termo dispõe de uma cláusula sobre a possível inclusão do caso para estudos científicos, resguardando sua identidade e seguindo os princípios éticos.

No momento do exame clínico, pôde-se visualizar que o elemento 11 já havia sido submetido a uma intervenção anterior, todos os aspectos clínicos extra-orais e intra-orais foram avaliados, tanto em tecido mole quanto tecido duro. Pode-se observar a presença de uma linha de fratura supragengivalmente na face vestibular e subgengivalmente na face palatina. Foi realizado teste de sensibilidade nos elementos superiores anteriores. No exame radiográfico, foi realizado o exame do tipo periapical com posicionador para avaliação da fratura. O plano de tratamento proposto para o elemento 11 foi pulpectomia e apicificação com Hidróxido de Cálcio. 
Concomitante ao relato do caso clínico, um levantamento bibliográfico na plataforma Pubmed, nos periódicos Dental Traumatology, Journal of Endodontics, International Endodontic Journal, Australian Dental Journal foi realizado, no qual os seguintes unitermos foram correlacionados: Apexification, Calcium Hydroxide, Open Apex, Imature Tooth, Dental Trauma. Inicialmente, foram artigos publicados entre 2001 e 2020. Somado aos artigos específicos, alguns autores fornecem suporte metodológicos: Estrela, C. (2018). Metodologia Científica: Ciência, Ensino, Pesquisa. Editora Artes Médicas; Pereira A. S. et al. (2018). Metodologia da pesquisa científica. [free e-book]. Santa Maria/RS. Ed. UAB/NTE/UFSM; Koche, J. C. (2011). Fundamentos de metodologia científica. Petrópolis: Vozes. Disponível; Ludke, M. \& Andre, M. E. D. A. (2013). Pesquisas em educação: uma abordagem qualitativa. São Paulo: E.P.U. Yin, R.K. (2015). O estudo de caso. Porto Alegre: Bookman.

\section{Relato de Caso}

Paciente do sexo masculino, 8 anos, foi encaminhado à clínica odontológica do curso de Especialização em endodontia do Ápice Cursos, 15 dias após sofrer queda da própria altura e trauma na região de incisivos superiores, com fratura do elemento 11 .

$\mathrm{Na}$ anamnese foi relatado que o paciente já foi atendido anteriormente, quando foi realizado acesso do elemento 11 e irrigação com $\mathrm{NaOCl} 1 \%$ (Solução de Milton). O dente foi deixado sem selamento e sem contenção, exposto na cavidade oral. Paciente fazia uso de medicação analgésica e sentia dor espontânea e ao manipular o fragmento dentário.

Durante o exame clínico nenhuma injúria foi detectada nos tecidos extraorais. O exame clínico intraoral revelou a presença de fratura oblíqua de coroa e raiz e mobilidade coronária do elemento 11, com exposição pulpar e presença de tecido de granulação proveniente do tecido gengival na linha de fratura. A linha de fratura estava localizada supragengivalmente na face vestibular (Fig.1) e subgengivalmente na face palatina. Foram realizados testes de sensibilidade ao frio com ar refrigerado (Endo Ice-Maquira. Paraná/ Brasil) nos elementos 12,11,21 e 22. Os dentes 12, 21 e 22 apresentaram resposta normal ao teste. O dente 11 apresentou ausência de resposta.

Ao exame radiográfico (Filme AGFA Dentus E-Speed- Kulzer. Hanau/ Alemanha) foi observada linha radiolúcida na região de terço cervical coronário, mas não pôde ser observada a linha de fratura em nível apical sugerindo fratura coronoradicular. Também pôde ser observada formação apical incompleta (Fig. 2).

Figura 1. Aspecto Clínico Inicial.

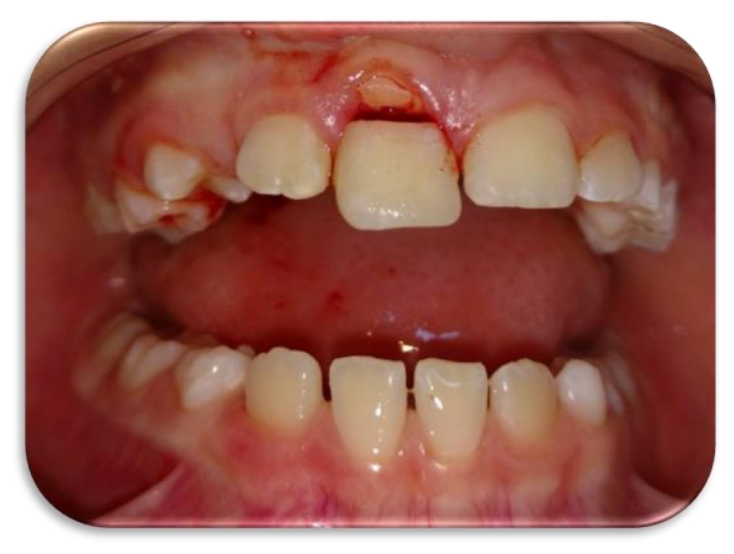

Fonte: Autores.

Figura 2. Radiografia periapical inicial demonstrando a linha de fratura cervical e a evidência de ápice aberto. 


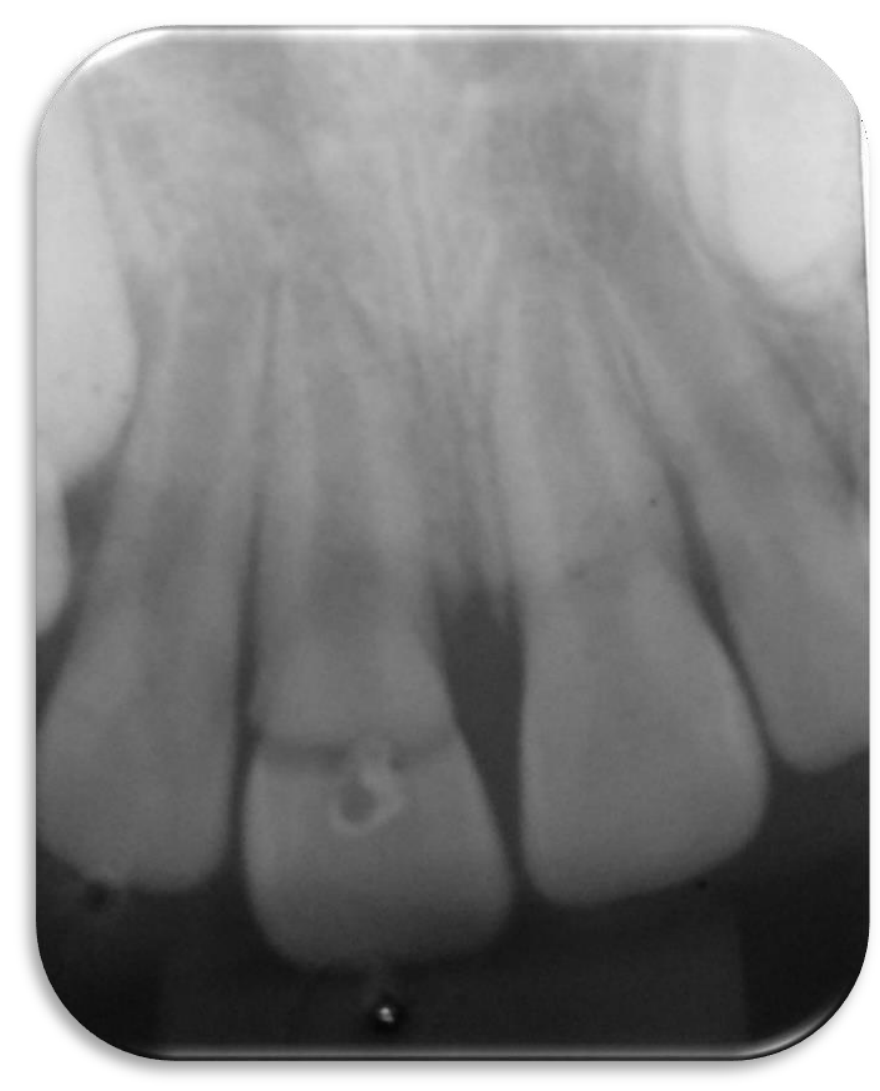

Fonte: Autores.

O elemento 11 foi diagnosticado com necrose pulpar, em decorrência do trauma. Levando em consideração a condição pulpar, a necessidade de reabilitação protética e as características de formação radicular incompleta, o plano de tratamento proposto para o elemento 11 foi pulpectomia e apicificação com Hidróxido de Cálcio.

Inicialmente foi realizada anestesia local com Alphacaine 2\% 1:100.000 (Nova DFL. Rio de Janeiro/ Brasil) técnica infiltrativa, remoção do tecido de granulação presente na linha de fratura, reposicionamento do fragmento coronário em justaposição ao fragmento apical e contenção do fragmento com resina composta (Fill Magic- Coltene. Altstätten/ Alemanha) (Fig. 3), abrangendo os elementos 12,11,21 e 22. Com o fragmento contido, foi realizado o refinamento do acesso à câmara pular, irrigação do canal com água destilada estéril e utilização de Clorexidina gel $2 \%$ como substância química auxiliar. A Clorexidina gel $2 \%$ também foi utilizada como medicação intracanal. O dente foi então selado provisoriamente com resina composta. 
Figura 3. Imobilização do fragmento dentário com resina composta.

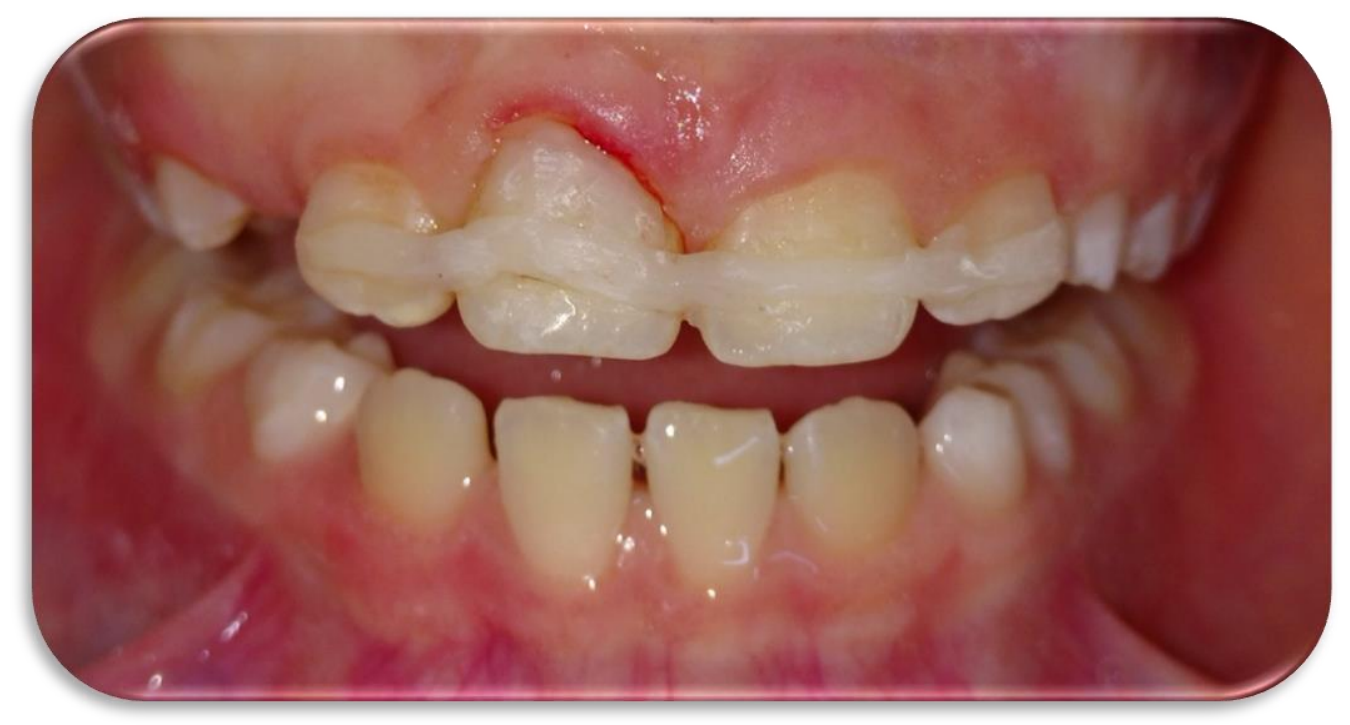

Fonte: Autores.

Na sessão subsequente foi realizada anestesia local com Alphacaine 2\% 1:100.000 técnica infiltrativa, isolamento absoluto utilizando grampo ( $N^{\circ}$ 209, Durflex- SS White. Rio de Janeiro/ Brasil) à distância no dente 14 e lençol de borracha (Sanctuary, K-Dent. São Paulo/ Brasil). Após o isolamento, o selamento provisório da coroa foi então removido e realizada odontometria com localizador apical (NovApex- Romidan. Rio de Janeiro/ Brasil) e lima K flex \#70 (ALLPRIME- Santa Catarina/ Brasil). A desinfecção foi realizada com gel de clorexidina 2\%, intercalando com irrigação de água destilada estéril e pincelamento das paredes dentinárias com lima rotatória 35/05 (ProDesign Logic, Easy do Brasil. Minas Gerais/ Brasil) 2mm aquém do comprimento obtido na odontometria. O canal foi irrigado com EDTA (Biodinâmica. Paraná/ Brasil) e a solução foi agitada utilizando Easy Clean (Easy do Brasil- Minas Gerais/ Brasil) para a remoção da camada de smear layer. Cones de papel calibrados em \#70 (Endo Tanari. Amazonas/ Brasil) foram utilizados para a secagem e a pasta de Hidróxido de Cálcio (Ultracal XS, Ultradent. Utah/ EUA) foi inserida com o auxílio de Navitip de $21 \mathrm{~mm}$ (Ultradent. Utah/ EUA) e mantida no canal durante 60 dias para a indução da formação de barreira mineralizada.

Passado o período de 60 dias e após a avaliação clínica e radiográfica deu-se início a obturação do canal. A pasta de hidróxido de cálcio foi removida através de irrigações alternadas de água destilada em seringa de $10 \mathrm{ml}$ (BD. Paraná/ Brasil) e agulha 0,55 x 20 mm (BD. Paraná/ Brasil) e Clorexidina gel 2\% como substância química auxiliar em seringa de $5 \mathrm{ml}$ (BD. Paraná/ Brasil) e agulha 0,55 x 20mm (BD. Paraná/ Brasil), agitando as soluções com Easy Clean. Foi observado que nessa segunda sessão a lima apical inicial ( $\mathrm{K}$ \#70) não conseguia atingir o forâme apical. Permanecendo $1 \mathrm{~mm}$ aquém da medida inicial obtida durante a odontometria, sinalizando uma diminuição do calibre apical inicial e dando condições à obturação do canal. Dessa forma, o EDTA foi a solução utilizada na última irrigação para a remoção de smear layer. O canal foi então seco com cones de papel calibrados em \#70 e obturado pela técnica do cone único com Guta Percha (FM Microtipped- (Endo Tanari. Amazonas/ Brasil) calibrada em \#70 e cimento endodôntico (Endomethasone N- Septodont. Lancaster/ EUA) (Fig. 4). 
Figura 4. Radiografia periapical final demonstrando a formação do ápice radicular evidenciada pela diminuição do calibre do forame apical.

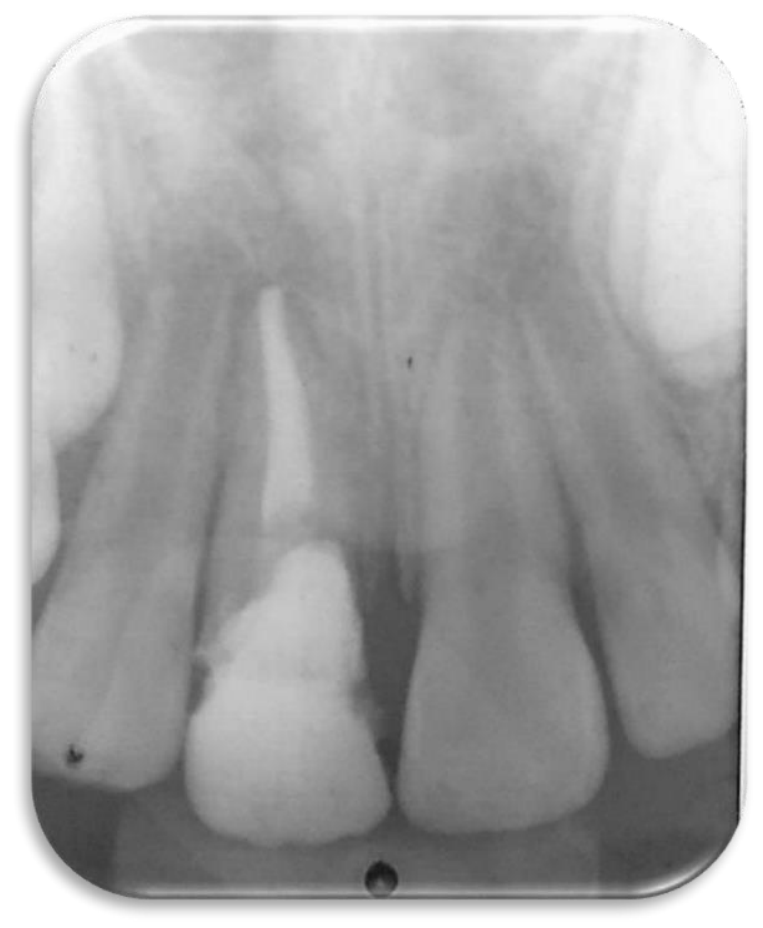

Fonte: Autores.

Na mesma sessão, foi selecionado o pino de fibra $N^{\circ} 2$ (WhitePost DC- FGM. Santa Catarina/ Brasil), realizada a desobturação parcial do canal para o espaço do pino e condicionamento do conduto com ácido fosfórico 37\% (AtacktecCaithec. Paraná/ Brasil) por 30 segundos. O ácido foi removido abundantemente e o conduto foi secado com cones de papel calibrados em \#70. O adesivo (Ambar- FGM. Santa Catarina/ Brasil) foi aplicado no interior do conduto e o excesso foi removido com microbrush (Cavibrush Longo- FGM. Santa Catarina/ Brasil) seco antes da polimerização por 20 segundos. A limpeza da superfície do pino com álcool $70 \%$ foi realizada, para então aplicar o silano (Prosil- FGM. Santa Catarina/ Brasil) e esperar a sua secagem natural. Passado esse tempo o adesivo (Ambar- FGM. Santa Catarina/ Brasil) foi aplicado em todo o pino e polimerizado. Para a finalização da cimentação o cimento resinoso (Allcem Core- FGM. Santa Catarina/ Brasil) foi misturado e inserido no interior do canal através da ponteira de automistura com bico aplicador conforme recomendação do fabricante. Deu-se início à polimerização de todas as faces dentárias. Após a cimentação foi instalada sobre o pino uma coroa provisória em resina acrílica (Fig. 5) e o paciente encaminhado para a confecção de coroa definitiva. 
Figura 5. Aspecto final após cimentação de coroa provisória.

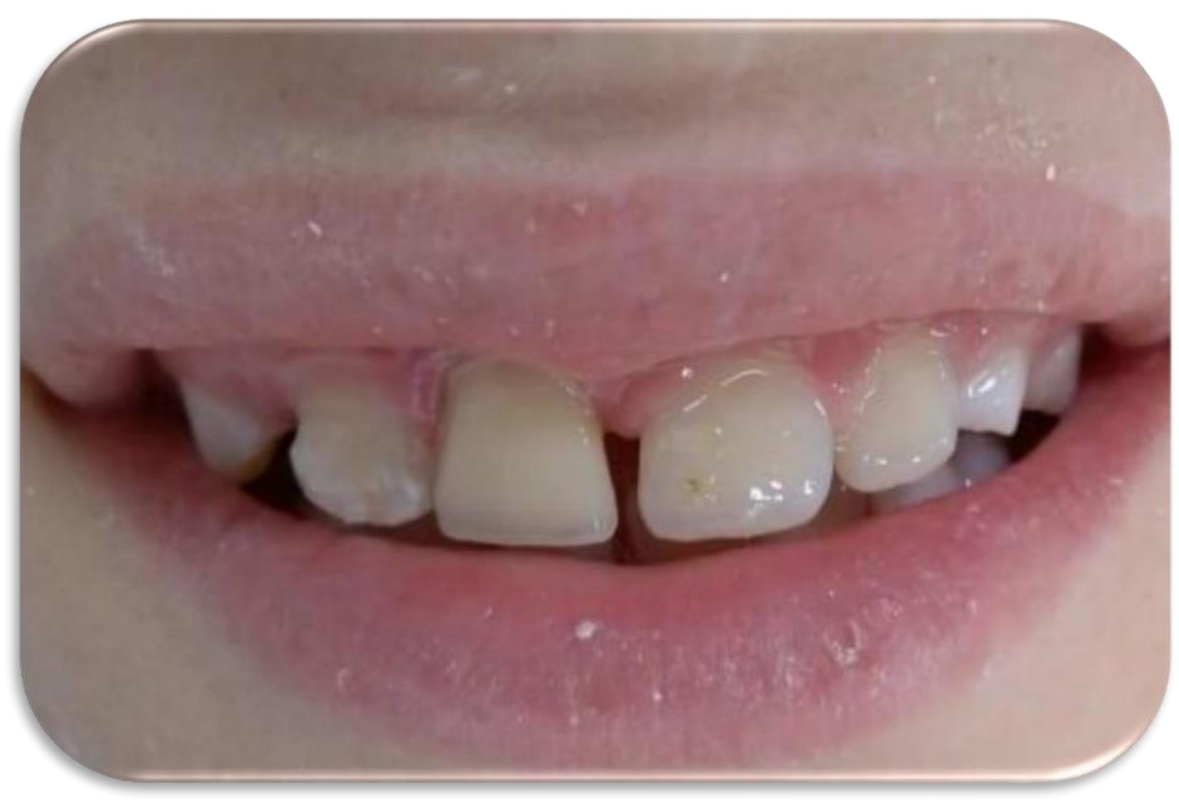

Fonte: Autores.

\section{Discussão}

O caso clínico relata o histórico de uma fratura corono-radicular, que é considerada uma consequência comum após trauma na região anterior da boca (Bajaj, Chordiya, Rudagi \& Patil, 2015). DiAngelis et al. (2012) recomendam que quando o trauma envolver um dente permanente imaturo, deve-se preconizar os procedimentos que permitam a manutenção da vitalidade pulpar. Entretanto, o caso descrito tem como status a necrose pulpar, o que impede a continuação do desenvolvimento radicular (Chala, Abouqal, \& Rida, 2011). A apicificação foi o tratamento de escolha justificado pela formação induzida de barreira calcificada a nível apical e reforço das paredes dentinárias, como descrito em trabalhos anteriores (Lopes, Siqueira Junior \& Estrela, 1999; Lee et al., 2014). A apicificação utilizando o hidróxido de cálcio para esse fim foi preconizada, por ser um material amplamente utilizado até os tempos atuais em razão da alta disponibilidade, fácil aplicação e baixo custo, além de haver uma maior quantidade de estudos que ajudam a prever o seu comportamento clínico. (Damle et al., 2016; Ince Yusufoglu, Ugur Aydin, Tulumbaci \& Bayrak, 2021; Shaik et al., 2021).

Além disso, a escolha do hidróxido de cálcio para o tratamento do referido caso, foi respaldada pela alta taxa de sucesso desse tratamento, assim como consta em diversos trabalhos, que demonstram a eficácia da utilização desta substância com taxa de sucesso variando de 74\% a 100\% (Alobaid, 2014; Damle et al., 2016; Dominguez Reyes, Munoz Munoz, \& Aznar Martin, 2005; Harlamb, 2016; Ince Yusufoglu, Ugur Aydin, Tulumbaci \& Bayrak, 2021; Kahler, Rossi-Fedele, Chugal \& Lin, 2017; Lin et al., 2016; Schmoldt, Kirkpatrick, Rutledge \& Yaccino, 2011). Dois fatores justificam a escolha do hidróxido de cálcio para o presente caso: $\mathrm{O}$ alto índice de sucesso que pode ser atribuído às propriedades do material, como $\mathrm{pH}$ alcalino $\mathrm{e}$ ação bactericida, além da habilidade de promover reparo e capacidade de formar barreira de tecido duro (Harlamb, 2016; Ince Yusufoglu, Ugur Aydin, Tulumbaci \& Bayrak, 2021). Segundo Bhola. Goyal, Tyagi e Kumar (2017), a combinação dessas propriedades cria um ambiente propício para a formação da barreira apical formada por osteocemento na região apical de um dente com ápice aberto, que é o objetivo primordial deste tratamento.

No caso clínico descrito, foram programadas sessões de trocas do hidróxido de cálcio. Entretanto, decorridos 60 dias de permanência sem trocas do material no interior do canal já foram observados a diminuição do diâmetro e fechamento apical. 
A obtenção do fechamento apical após uma única aplicação do material pode ser explicada pelo fato do hidróxido de cálcio ser necessário apenas para a reação inicial de cura, sendo necessária uma única aplicação assim como relatado por Vellore (2010). A reposição de hidróxido de cálcio é recomendada quando há o desenvolvimento de sintomas ou quando o material apresenta falhas radiograficamente detectáveis (Vellore, 2010) o que não ocorreu no caso clínico descrito.

A permanência do hidróxido de cálcio por apenas 60 dias corrobora com o tempo descrito por Valera et al. (2015), e contribuiu para o sucesso do tratamento, visto que o tempo de tratamento prolongado é considerado uma desvantagem, que pode levar a reinfecção do canal através de infiltrações coronárias, aumentar as chances de fratura e levar à desistência do tratamento pelo paciente, como descrito em outros trabalhos (Bhola. Goyal, Tyagi, \& Kumar, 2017; Kahler, Rossi-Fedele, Chugal \& Lin, 2017). Embora o tempo de 60 dias preconizado no presente caso tenha sido satisfatório, a literatura demonstra tempos variáveis de permanência do hidróxido de cálcio, 2 a 12 meses (Valera et al., 2015); 12,19 meses (Dominguez Reyes, Munoz Munoz, \& Aznar Martin, 2005); 13 a 67 semanas (Vellore, 2010); 20 meses (Soares et al., 2012 ); 3 a 24 meses (Damle et al., 2016; Shabahang, 2013); 3 a 17 meses (Silveira, Sebrão, Vilanova \& Sánchez-Ayala, 2015); 5 a 20 meses (Lin et al., 2016) e 12,6 meses (Kahler, Rossi-Fedele, Chugal \& Lin, 2017).

Levando em consideração a maior susceptibilidade dos dentes permanentes imaturos à fratura devido à formação incompleta, assim como relatado nos trabalhos de Schmoldt, Kirkpatrick, Rutledge e Yaccino (2011) e Kahler, Rossi-Fedele, Chugal e Lin (2017), no presente caso, o preparo químico mecânico preconizou o desgaste mínimo das paredes radiculares. A instrumentação empregada $2 \mathrm{~mm}$ aquém do comprimento obtido na odontometria, como descrito no caso, teve como objetivo evitar a agressão dos remanescentes da bainha radicular de Hertwig e da papila apical, o que segundo Harlamb (2016) e Prompreecha, Sastraruji, Louwakul e Srisuwan (2018) levaria à cessação da formação adicional do ápice radicular. Essa limitação na remoção mecânica de microrganismos em dentes imaturos gera uma dependência maior da ação química promovida pelos irrigantes e medicações utilizadas durante o tratamento endodôntico.

Apesar da maioria dos autores optar pela ação química promovida pela irrigação com hipoclorito (Bajaj, Chordiya, Rudagi \& Patil, 2015; Bhola. Goyal, Tyagi, \& Kumar, 2017; Dominguez Reyes, Munoz Munoz, \& Aznar Martin, 2005; Fonzar, Forner, Fabian-Fonzar \& Llena, 2018; Harlamb, 2016; Kubo et al., 2018; Lee et al., 2014; Soares et al., 2012; Valera et al., 2015), no caso clínico descrito o gel de clorexidina 2,0\% foi escolhido como solução química auxiliar assim como nos casos de Lee, Hsiao, Chang e Chen (2010), Soares et al. (2012) e Prompreecha, Sastraruji, Louwakul e Srisuwan (2018). Essa escolha foi baseada na priorização da segurança e menor dano aos tecidos periapicais e células da papila apical, além da desinfecção adequada (Lee, Hsiao, Chang \& Chen, 2010; Soares et al., 2012). Além disso, o hipoclorito é uma substância tóxica aos tecidos periapicais, capaz de modificar a matriz dentinária e reduzir a resistência à fratura (Valera et al., 2015), fato que deve ser evitado durante o tratamento de dentes imaturos.

A fratura corono-radicular causada pelo trauma se estendeu ao nível subgengival, tornando o procedimento reabilitador um maior desafio. A reabilitação do dente acometido levou em consideração a fragilidade inerente ao dente imaturo e a consequente necessidade de reforço das paredes radiculares. Optou-se então pela instalação do pino de fibra de vidro, uma vez que a resistência à flexão dos pinos de fibra se assemelha muito à da dentina (Kubo et al., 2018; Schmoldt, Kirkpatrick, Rutledge \& Yaccino, 2011). Esta qualidade pode fornecer maior resistência à fratura de raízes imaturas frágeis, enquanto diminui a incidência de fratura.

Levando-se em consideração as características anatômicas da coroa dental de um paciente jovem, foi planejada a instalação de uma coroa protética fixa que deverá ser trocada à medida que as características da dentição forem se modificando com o passar da idade do paciente. Apesar de se tratar de uma criança sujeita a sofrer novos traumas nessa região, o caso apresentou um resultado satisfatório e permitiu a reabilitação funcional e estética do paciente, permitindo-o voltar às suas atividades habituais. Os responsáveis foram orientados aos retornos periódicos de acompanhamento radiográfico após 3 meses, 
6 meses e 1 ano após a finalização do caso. Porém não retornaram para as consultas que haviam sido planejadas.

\section{Considerações Finais}

O tratamento de dentes imaturos traumatizados é normalmente complexo e de difícil execução até para os endodontistas mais experientes. Diante dessas dificuldades, torna-se imprescindível ao profissional dispor de técnicas que ao mesmo tempo permitam o restabelecimento funcional e estético do paciente, e que sejam viáveis e de fácil execução. A apicificação utilizando hidróxido de cálcio demonstrou ser uma técnica eficaz no tratamento de dentes imaturos com ápice aberto ao induzir a deposição mineral e consequente continuação do desenvolvimento do ápice radicular. Por se tratar de uma técnica consolidada na literatura, de baixo custo e fácil execução, o emprego da apicificação utilizando hidróxido de cálcio atingiu seu principal objetivo, que é criar condições para a adaptação dos materiais durante a obturação e assim permitir a reabilitação dental. A técnica utiliza materiais presentes na rotina dos endodontistas e tem alta reprodutibilidade, podendo ser facilmente inserido nos planos de tratamento para dentes permanentes com a evidência de ápice aberto. Para melhoria de protocolos e para que se tornem mais acessíveis, mais estudos fazem-se necessários acerca da engenharia tecidual baseada em células-tronco, afim de que tornem-se mais viáveis para a prática clínica.

\section{Referências}

Alobaid, A. S., Cortes, L. M., Lo, J., Nguyen, T. T., Albert, J., Abu-Melha, A. S., Gibbs, J. L. (2014). Radiographic and clinical outcomes of the treatment of immature permanent teeth by revascularization or apexification: a pilot retrospective cohort study. Journal of endodontics. 40(8), 1063-1070.

Bajaj, P., Chordiya, R., Rudagi, K., \& Patil, N. (2015). Multidisciplinary approach to the management of complicated crown-root fracture: a case report. Journal of international oral health: JIOH. 7(4), 88.

Bhola, M., Goyal, V., Tyagi, P., \& Kumar, T. (2017). Mineral trioxide aggregate-induced apical closure in nonvital immature permanent maxillary incisor. Journal of Indian Society of Pedodontics and Preventive Dentistry. 35(4), 378.

Botero, T. M., Tang, X., Gardner, R., Hu, J. C., Boynton, J. R., \& Holland, G. R. (2017). Clinical evidence for regenerative endodontic procedures: immediate versus delayed induction? Journal of endodontics. 43(9), S75-S81.

Boufdil, H., Mtalsi, M., El Arabi, S., \& Bousfiha, B. (2020). Apexification with Calcium Hydroxide vs. Revascularization. Case reports in dentistry.

Chala, S., Abouqal, R., \& Rida, S. (2011). Apexification of immature teeth with calcium hydroxide or mineral trioxide aggregate: systematic review and metaanalysis. Oral Surgery, Oral Medicine, Oral Pathology, Oral Radiology, and Endodontology. 112(4), e36-e42.

Damle, S. G., Bhattal, H., Damle, D., Dhindsa, A., Loomba, A., \& Singla, S. (2016). Clinical and radiographic assessment of mineral trioxide aggregate and calcium hydroxide as apexification agents in traumatized young permanent anterior teeth: A comparative study. Dental research jornal. 13(3), 284.

Jesus Soares, A., Nagata, J. Y., Casarin, R. C. V., de Almeida, J. F. A., de Almeida Gomes, B. P. F., Zaia, A. A., de Souza-Filho, F. J. (2012). Apexification with a new intra-canal medicament: a multidisciplinary case report. Iranian Endodontic Journal. 7(3), 165.

DiAngelis, A., Andreasen, J. O., Ebeleseder, K. A., Kenny, D. J., Trope, M., Sigurdsson A., et al. (2012) International Association of dental Traumatology guidlines for the management of traumatic dental injuries:1, Fractures and luxations of permanente teeth. Dent Traumatol. 28 , 2-12.

Dominguez Reyes, A., Munoz, L., \& Aznar Martin, T. (2005). Study of calcium hydroxide apexification in 26 young permanent incisors. Dental Traumatology. 21(3), 141-145.

Fonzar, F., Forner, L., Fabian-Fonzar, R., \& Llena, C. (2018). Induced post-traumatic apexification: 20 year follow-up and morphological study after new fracture. Annals of Anatomy-Anatomischer Anzeiger. 216, 120-124.

Harlamb, S. C. (2016). Management of incompletely developed teeth requiring root canal treatment. Australian dental jornal. 61, 95-106.

Ince Yusufoglu, S., Ugur Aydin, Z., Tulumbaci, F., \& Bayrak, S. (2021). Evaluation of different Apexification treatments of teeth with immature apices and apical periodontitis on the fractal dimensions of trabecular bone. Australian Endodontic Journal. 47(2), 163-169.

Kahler, B., Rossi-Fedele, G., Chugal, N., \& Lin, L. M. (2017). An evidence-based review of the efficacy of treatment approaches for immature permanent teeth with pulp necrosis. Journal of endodontics. 43(7), 1052-1057.

Kandemir Demirci, G., Kaval, M. E., Güneri, P., \& Çalışkan, M. K. (2020). Treatment of immature teeth with nonvital pulps in adults: a prospective comparative clinical study comparing MTA with $\mathrm{Ca}(\mathrm{OH}) 2$. International endodontic jornal. 53(1), 5-18.

Kubo, M., Komada, W., Otake, S., Inagaki, T., Omori, S., \& Miura, H. (2018). The effect of glass fiber posts and ribbons on the fracture strength of teeth with flared root canals restored using composite resin post and cores. Journal of prosthodontic research. 62(1), 97-103. 
Lee, L. W., Hsieh, S. C., Lin, Y. H., Huang, C. F., Hsiao, S. H., \& Hung, W. C. (2015). Comparison of clinical outcomes for 40 necrotic immature permanent incisors treated with calcium hydroxide or mineral trioxide aggregate apexification/apexogenesis. Journal of the Formosan Medical Association. 114(2), 139146.

Lee, L. W., Hsiao, S. H., Chang, C. C., \& Chen, L. K. (2010). Duration for apical barrier formation in necrotic immature permanent incisors treated with calcium hydroxide apexification using ultrasonic or hand filing. Journal of the Formosan Medical Association. 109(8), 596-602.

Lin, J. C., Lu, J. X., Zeng, Q., Zhao, W., Li, W. Q., \& Ling, J. Q. (2016). Comparison of mineral trioxide aggregate and calcium hydroxide for apexification of immature permanent teeth: A systematic review and meta-analysis. Journal of the Formosan Medical Association. 115(7), 523-530.

Lopes, H. P., Siqueira Junior, J. F., \& Estrela, C. (1999). Tratamento endodôntico em dentes com rizogênese incompleta. In Endodontia: biologia e técnica (pp. 485-96).

Lopes J. H. P., Siqueira Jr. J. F. \& Neves M.A.S. (2015) Tratamento Endodôntico de Dentes com Rizogênese Incompleta. In: Endodontia Biologia e Técnica. $4^{\mathrm{a}}$ Ed. Elsevier. P.1456-1479.

Prompreecha, S., Sastraruji, T., Louwakul, P., \& Srisuwan, T. (2018). Dynamic irrigation promotes apical papilla cell attachment in an ex vivo immature root canal model. Journal of endodontics. 44(5), 744-750.

Refaei, P., Jahromi, M. Z., \& Moughari, A. A. K. (2020). Comparison of the microleakage of mineral trioxide aggregate, calcium-enriched mixture cement, and Biodentine orthograde apical plug. Dental research jornal. 17(1), 66.

Schmoldt, S. J., Kirkpatrick, T. C., Rutledge, R. E., \& Yaccino, J. M. (2011). Reinforcement of simulated immature roots restored with composite resin, mineral trioxide aggregate, gutta-percha, or a fiber post after thermocycling. Journal of endodontics. 37(10), 1390-1393.

Shabahang, S. (2013). Treatment options: apexogenesis and apexification. Pediatric dentistry. 35(2), 125-128.

Shaik, I., Dasari, B., Kolichala, R., Doos, M., Qadri, F., Arokiyasamy, J. L., \& Tiwari, R. (2021). Comparison of the Success Rate of Mineral Trioxide Aggregate, Endosequence Bioceramic Root Repair Material, and Calcium Hydroxide for Apexification of Immature Permanent Teeth: Systematic Review and Meta-Analysis. Journal of pharmacy \& bioallied sciences. 13(Suppl 1), S43-S47

Silveira, C. M. M., Sebrão, C. C. N., Vilanova, L. S. R., \& Sánchez-Ayala, A. (2015). Apexification of an immature permanent incisor with the use of calcium hydroxide: 16-year follow-up of a case. Case reports in dentistry.

Valera, M. C., Albuquerque, M. T. P., Yamasaki, M. C., Vassallo, F. N. S., da Silva, D. A. E. S. A., \& Nagata, J. Y. (2015). Fracture resistance of weakened bovine teeth after long-term use of calcium hydroxide. Dental Traumatology. 31(5), 385-389.

Vellore, K. G. (2010). Calcium hydroxide induced apical barrier in fractured nonvital immature permanent incisors. Journal of Indian Society of Pedodontics and Preventive Dentistry. 28(2), 110.

Zengin, A. Z., Celenk, P., Sumer, A. P., \& Cankaya, S. (2015). Evaluation of traumatic dental injuries in a group of Turkish population. Nigerian Journal of clinical practice. $18(1), 86-89$. 